\title{
On the Optimum Number of Hypotheses for Adaptive Reduced-Rank Subspace Selection
}

\author{
Markus Hofer ${ }^{1}$, Zhinan $\mathrm{Xu}^{1}$, Thomas Zemen ${ }^{1,2}$ \\ ${ }^{1}$ FTW Forschungszentrum Telekommunikation Wien, Vienna, Austria \\ ${ }^{2}$ AIT Austrian Institute of Technology, Vienna, Austria \\ Email: \{markus.hofer, xu\}@ftw.at, thomas.zemen@ait.ac.at
}

\begin{abstract}
Intelligent transport systems (ITS) require lowlatency dependable wireless communication links inbetween vehicles as well as between vehicles and the infrastructure. In vehicular communication scenarios communication channels are time- and frequency (doubly) dispersive and the channel statistics are non-stationary, i.e., they change over time. Hence, the design of appropriate channel estimators is challenging. Recently an adaptive reduced-rank channel estimation technique for nonstationary time-variant channel estimation was introduced by Zemen and Molisch, 2012. This technique uses a hypothesis test to obtain an estimate of the current channel statistics on a per frame basis. The optimum number of hypotheses is not known. In this paper we present new empirical insights on the optimum choice of the number of hypotheses for the hypothesis test for non-stationary time-variant channel estimation. With these considerations the complexity of an adaptive reduced-rank channel estimator can be reduced and its performance improved.
\end{abstract}

\section{INTRODUCTION}

Low-latency dependable wireless vehicle-to-vehicle (V2V) communication is a crucial building block for future intelligent transport systems (ITSs) [1] to reduce the number of casualties on the road. For ITS different access technologies are under consideration [2] where besides 802.11p [3] also 3GPP long term evolution (LTE) [4] is recently investigated as potential candidate [5]

Reliable vehicular communication requires channel estimation methods that can deal with high delay- and Doppler spreads in non-stationary propagation conditions, i.e., where the delay and Doppler spread changes over time [6]. Such propagation conditions are specifically observed in V2V communication scenarios [7-10].

In $[11,12]$ a robust reduced-rank iterative channel estimator that relies on a correlation matrix assuming a flat delayDoppler scattering function is presented. The support region of the scattering function is defined by the expected maximum delay and the maximum Doppler frequency of the channel in a certain communication scenario. The eigenvectors of the channel's autocorrelation matrix can be approximated by a two dimensional subspace model using generalized discrete prolate spheroidal (DPS) sequences [13, 14]. Such an approach is mismatched to the actual support of the scattering function.

To improve the performance, an adaptive subspace selection algorithm that estimates the actual delay and Doppler support of the current channel realization was presented in [6] for the pilot pattern of IEEE 802.11p [3] and in [15] for the pilot pattern of the LTE downlink. The adaptive subspace selection algorithm [6] is based on the work of [16] and utilizes a frame based hypothesis test that uses the observations at pilot positions to obtain an estimate on the delay and Doppler support of the current channel realization. For the hypothesis test the optimum number of hypotheses that provides the best estimation performance is not known. In this paper we consider pilot based adaptive reduced-rank channel estimation for time-variant frequency-flat channels, i.e. a single subcarrier in an orhtogonal frequency division multiplexing (OFDM) system.

Contributions of the Paper: (a) We present a search algorithm for the optimum number of hypotheses for a timeselective channel. (b) A hypotheses design is given that provides a good trade-off between estimation performance and complexity. These results allow to reduce both, the estimation error and the complexity of an adaptive reduced-rank channel estimator.

The remainder of this paper is organized as follows: In Section II we introduce the system model for time-variant frequency-flat channel estimation. We briefly discuss the hypothesis test and present the framework for the optimization of the number of hypotheses. In Section III we present simulations results and discuss the obtained results. Finally, in Section IV we conclude the presented work.

\section{System ModeL}

\section{A. Time-Variant Frequency-Flat Channel}

We consider the transmission of a symbol sequence $d[m]$ of length $M$ over a time-variant frequency-flat fading channel $h(t)$. The discrete time received signal sequence $y[m]$ can be modeled by

$$
y[m]=d[m] h[m]+z[m],
$$

where $m \in\{0, \ldots, M-1\}$ denotes the discrete time index, $h[m]=h\left(m T_{s}\right)$ the sampled channel, $T_{s}$ the symbol duration and $z[m] \sim \mathcal{C N}\left(0, \sigma_{z}^{2}\right)$ the symmetric complex additive white Gaussian noise with zero mean and variance $\sigma_{z}^{2}$, respectively. The symbol sequence $d[m]$ consists of $M-N_{p}$ data symbols $b[m]$ interleaved with $N_{p}$ pilot symbols $p[m]$

$$
d[m]=b[m]+p[m] .
$$

The data and pilot symbols are drawn from a QPSK symbol alphabet and $b[m]=0$ for $m \in \mathcal{P}$. The pilot placement is 
defined by the index set

$$
\mathcal{P}=\left\{k \Delta_{t}: k \in\left\{0,1, \ldots, N_{p}-1\right\}\right\},
$$

where $\Delta_{t}$ represent the pilot symbol distance. Defining the length $M$ received column vector $\mathbf{y} \in \mathbb{C}^{M \times 1}$ by $[\mathbf{y}]_{m+1}=$ $y[m], m=0, \ldots, M-1$ we can rewrite (1) in matrix-vector form according to

$$
\mathbf{y}=\mathbf{D h}+\mathbf{z} .
$$

The length $M$ vectors $\mathbf{d} \in \mathbb{C}^{M \times 1}, \mathbf{h} \in \mathbb{C}^{M \times 1}$, and $\mathbf{z} \in \mathbb{C}^{M \times 1}$ are defined similarly to $y$ and contain the transmitted symbols, the channel coefficients and the noise samples, respectively. The matrix $\mathbf{D}=\operatorname{diag}(\mathbf{d})$ is a diagonal matrix of size $M \times M$ with the elements of $\mathbf{d}$ on its diagonal.

To estimate the unknown channel vector $\mathbf{h}$ we use a linear minimum mean square error (LMMSE) estimator

$$
\hat{\mathbf{h}}_{\text {LMMSE }}=\mathbf{R}_{h ; h_{p}} \mathbf{D}_{p}^{H}\left(\mathbf{D}_{p} \mathbf{R}_{h_{p}} \mathbf{D}_{p}^{H}+\sigma_{z}^{2} \mathbf{I}_{N_{p}}\right)^{-1} \mathbf{y}_{p}
$$

that provides the channel estimate $\hat{\mathbf{h}}_{\mathrm{LMMSE}}$. Here $\mathbf{I}_{N_{p}}$ represents the identity matrix of size $N_{p} \times N_{p}$ and $(\cdot)^{H}$ denotes the conjugate transpose. The vectors $\mathbf{y}_{p} \in \mathbb{C}^{N_{p} \times 1}, \mathbf{h}_{p} \in \mathbb{C}^{N_{p} \times 1}$, and $\mathbf{n}_{p} \in \mathbb{C}^{N_{p} \times 1}$ contain the noisy observations, the channel, and the noise samples, respectively, at pilot positions only. They are given by $\left[\mathbf{y}_{p}\right]_{k+1}=y\left[k \Delta_{t}\right],\left[\mathbf{h}_{p}\right]_{k+1}=h\left[k \Delta_{t}\right]$, and $\left[\mathbf{z}_{p}\right]_{k+1}=z\left[k \Delta_{t}\right]$ for $k=0, \ldots, N_{p}-1$, respectively. The matrix $\mathbf{D}_{p}=\operatorname{diag}\left(\mathbf{d}_{p}\right)$ is of size $N_{p} \times N_{p}$ and $\mathbf{d}_{p} \in \mathbb{C}^{N_{p} \times 1}$ is the vector of pilot symbols. Furthermore, $\mathbf{R}_{h_{p}}=\mathbb{E}\left\{\mathbf{h}_{p} \mathbf{h}_{p}^{H}\right\}$ is the autocorrelation matrix of the channel at pilot positions and $\mathbf{R}_{h, h_{p}}=\mathbb{E}\left\{\mathbf{h h}_{p}^{H}\right\}$ is the cross correlation matrix between the channel at all positions and the channel at pilot positions. They can be obtained as sub-matrices of the channel autocorrelation matrices $\mathbf{R}_{h}=\mathbb{E}\left\{\mathbf{h} \mathbf{h}^{H}\right\}$ by extracting $\Delta_{t}$-spaced rows and/or columns. See [17] for further details.

\section{B. Review of Hypothesis Test}

The autocorrelation matrix $\mathbf{R}_{h}$ is in general not known to the receiver and has to be estimated from the channel observations. Direct estimation of the autocorrelation matrix from a single observation would lead to high estimation errors due to the short frame length and the low duty cycle of ITS data traffic [6, 14]. Advanced estimators [18] do not consider the case when the number of observed realizations of a statistical process is smaller than the observation dimension. Therefore a hypothesis test is utilized. The technique of hypothesis testing is based on the subspace selection algorithm of Beheshti et al. presented in [16]. We will briefly review the principle of hypothesis testing for the time-variant frequencyflat channel and refer the reader to [6, 14-16] for more detailed information on hypothesis testing for time-variant frequencyselective channels. In $[6,15]$ especially the orthogonal frequency division multiplexing (OFDM) based systems $802.11 \mathrm{p}$ and LTE are considered.

To estimate the autocorrelation matrix $\mathbf{R}_{h}$ we firstly approximate it by a robust autocorrelation matrix $\tilde{\mathbf{R}}_{h}$ that assumes a flat Doppler power spectral density (DSD) within the support region

$$
W_{t}=\left[-\nu_{\mathrm{D}}, \nu_{\mathrm{D}}\right],
$$

with $0 \leq \nu_{\mathrm{D}} \leq \nu_{\text {Dmax }}$. Here $\nu_{\text {Dmax }}$ represents the maximum normalized one sided Doppler bandwidth of the channel. The approximation of a Clarke's Doppler spectrum with a flat DSD causes only a minor degradation in estimation performance [6]. We assume that the fading process is wide-sense stationary within the considered observation region [9]. The normalized Doppler bandwidth is definedy by $\nu_{\mathrm{D}}=f_{\mathrm{D}} T_{s}$, where $f_{\mathrm{D}}=$ $v / c_{0} f_{c}$, with $v$ the relative velocity between transmitter and receiver, $c_{0}$ the speed of light and $f_{c}$ the carrier frequency of the system. For an autocorrelation matrix with flat DSD the eigenvectors $\mathbf{U}\left(W_{t}, I_{t}\right)$ of

$$
\tilde{\mathbf{R}}_{h}\left(W_{t}, I_{t}\right)=\mathbf{U}\left(W_{t}, I_{t}\right) \boldsymbol{\Sigma}\left(W_{t}, I_{t}\right) \mathbf{U}\left(W_{t}, I_{t}\right)^{H}
$$

are also given by the DPS [13] sequences where $\boldsymbol{\Sigma}\left(W_{t}, I_{t}\right)$ is the diagonal matrix of eigenvalues $\lambda_{i}\left(W_{t}, I_{t}\right)$ and $I_{t}=$ $[0, \ldots, M-1]$ is the finite index set where the fading process is observed on. The eigenvalues $\lambda_{i}\left(W_{t}, I_{t}\right)$ are sorted in descending order, i.e., $\lambda_{0}\left(W_{t}, I_{t}\right) \geq \lambda_{1}\left(W_{t}, I_{t}\right) \geq \ldots \lambda_{M-1}\left(W_{t}, I_{t}\right)$ and quickly decay for $i>D^{\prime}$ with $D^{\prime}\left(W_{t}, I_{t}\right)=\left\lceil\left|W_{t}\right| M\right\rceil+1$. This allows us to approximate the autocorrelation matrix $\tilde{\mathbf{R}}_{h}$ by a robust reduced-rank matrix $\tilde{\mathbf{R}}_{h} \approx \breve{\mathbf{R}}_{h}=\mathbf{U}_{D} \boldsymbol{\Sigma}_{D} \mathbf{U}_{D}^{H}$ with $D$ dominant eigenvalues, where $\mathbf{U}_{D}$ and $\boldsymbol{\Sigma}_{D}$ contain the first $D$ coloumns of $\mathbf{U}$ and $\boldsymbol{\Sigma}$, respectively. The dimension $D$ for a given noise variance $\sigma_{z}^{2}$ is found to be [19]

$$
D=\underset{\mathcal{D} \in\{1, \ldots, M\}}{\operatorname{argmin}}\left(\frac{1}{M} \sum_{i=\mathcal{D}}^{M-1} \lambda_{i}\left(W_{t}, I_{t}\right)+\frac{\mathcal{D}}{M} \sigma_{z}^{2}\right) .
$$

Note that (8) depends on the noise variance $\sigma_{z}^{2}$. Furthermore, the dimension $D$ is only optimal in the case where $\mathbf{R}_{h}=\tilde{\mathbf{R}}_{h}$. In any other case we get a (slightly) suboptimal dimension.

The goal of the hypothesis test is to estimate the support of the DSD $W_{t}=\left[-\nu_{\mathrm{D}}, \nu_{\mathrm{D}}\right] \subset\left[-\nu_{\mathrm{Dmax}}, \nu_{\text {Dmax }}\right]$, that defines the autocorrelation matrix $\mathbf{R}_{h}$, from the observations at pilot positions $\mathcal{P}$. The signal model at pilot positions is given by

$$
\mathbf{y}_{p}=\mathbf{D}_{p} \mathbf{h}_{p}+\mathbf{z}_{p}
$$

with the vectors $\mathbf{y}_{p}, \mathbf{z}_{p}$ and the matrix $\mathbf{D}_{p}$ defined in Section II-A. The channel observations at the pilot positions $\mathcal{P}$ are obtained by

$$
\mathbf{w}_{p}=\mathbf{D}_{p}^{H} \mathbf{y}_{p}=\mathbf{h}_{p}+\mathbf{D}_{p}^{H} \mathbf{z}_{p}=\mathbf{h}_{p}+\mathbf{z}_{p}^{\prime},
$$

where $\mathbf{z}_{p}^{\prime} \sim \mathcal{C N}\left(0, \sigma_{z}^{2} \mathbf{I}_{N_{p}}\right)$ has the same statistics as $\mathbf{z}_{p}$. The fading process observed at the pilot positions is described by the eigenvectors of the autocorrelation matrix $\mathbf{R}_{h_{p}}$. For an equidistant pilot grid the eigenvectors $\mathbf{U}^{(\mathcal{P})}\left(W_{t}, \mathcal{P}\right)$ of a fading process $\tilde{\mathbf{R}}_{h_{p}}$ that assumes a flat DSD are also spanned by sampled DPS sequences [15]. For the setup of $\mathbf{U}^{(\mathcal{P})}$ we use [6, Eq. (20)-(25)] and set $\nu_{\mathrm{D}}^{\prime}=\Delta_{t} \nu_{\mathrm{D}}$ [15]. From this setup we obtain $\boldsymbol{\Sigma}^{(\mathcal{P})}$. To determine the dimension $D^{(\mathcal{P})}$ we use a slight variation of (8)

$$
D^{(\mathcal{P})}=\underset{\mathcal{D}^{(\mathcal{P})} \in\left\{1, \ldots, N_{p}\right\}}{\operatorname{argmin}}\left(\frac{1}{N_{p}} \sum_{i=\mathcal{D}^{(\mathcal{P})}}^{N_{p}-1} \lambda_{i}^{(\mathcal{P})}\left(W_{t}, \mathcal{P}\right)+\frac{\mathcal{D}^{(\mathcal{P})}}{N_{p}} \sigma_{z}^{2}\right),
$$


where $\lambda_{i}^{(\mathcal{P})}\left(W_{t}, \mathcal{P}\right)$ are the eigenvalues of the fading process $\tilde{\mathbf{R}}_{h_{p}}$.

For the hypothesis test we define a finite set of $A$ hypotheses $\left\{W_{t}(1), \ldots, W_{t}(a), \ldots, W_{t}(A)\right\}$ on the DSD support where each hypothesis has a different support

$$
W_{t}(a)=\left(-\frac{a}{A} \cdot \nu_{\text {Dmax }}, \frac{a}{A} \cdot \nu_{\text {Dmax }}\right),
$$

$a \in\{1, \ldots, A\}$. Each hypothesis represents a subspace spanned by the columns of $\mathbf{U}\left(W_{t}(a), I_{t}\right)$ which corresponds to the subspace $\mathbf{U}^{(\mathcal{P})}\left(W_{t}(a), \mathcal{P}\right)$ at pilot positions. The hypothesis test provides the hypothesis $\hat{a} \in\{1, \ldots, A\}$ that, according to the test, fits best to the current channel realization. For the sake of simplicity of notation we substitute $\mathbf{U}^{(\mathcal{P})}\left(W_{t}(a), \mathcal{P}\right)$ by $\mathbf{U}_{a}^{(\mathcal{P})}$ in the following. We calculate the channel estimates on the pilot positions for each hypothesis according to $[6,16]$

$$
\hat{\mathbf{h}}_{p}(a)=\mathbf{U}_{a}^{(\mathcal{P})} \mathbf{U}_{a}^{(\mathcal{P})}{ }^{H} \mathbf{w}_{p} .
$$

The data error for hypothesis $a$ can then be expressed by

$$
x_{a}=\frac{1}{N_{p}}\left\|\mathbf{w}_{p}-\hat{\mathbf{h}}_{p}(a)\right\|^{2} .
$$

However, the metric that we want to minimize is not the data error $x_{a}$ but the reconstruction error

$$
z_{a}=\frac{1}{N_{p}}\left\|\mathbf{h}_{p}-\hat{\mathbf{h}}_{p}(a)\right\|^{2}
$$

which cannot be directly observed at the receiver side since the actual channel $\mathbf{h}_{p}$ is not known to the receiver. Knowing $x_{a}$, we are interested to obtain a probabilistic upper bound on $z_{a}$ as

$$
z_{a}<\overline{z_{a}}\left(x_{a}, p_{1}, p_{2}\right)
$$

that only depends on $x_{a}$ and some constants $p_{1}$ and $p_{2}$. This bound enables us to select the best hypothesis $W_{t}(\hat{a})$, i.e.,

$$
\hat{a}=\underset{a \in\{1, \ldots, A\}}{\operatorname{argmin}} \overline{z_{a}}\left(x_{a}, p_{1}, p_{2}\right) .
$$

With the hypothesis $W_{t}(\hat{a})$ and (7) we obtain $\tilde{\mathbf{R}}_{h}^{\hat{a}} \approx \breve{\mathbf{R}}_{h}^{\hat{a}}$ of the current channel realization that is used to obtain the channel estimate $\hat{\mathbf{h}}_{\text {LMMSE }}^{\hat{a}}$ according to

$$
\hat{\mathbf{h}}_{\mathrm{LMMSE}}^{\hat{a}}=\tilde{\mathbf{R}}_{h ; h_{p}}^{\hat{a}} \mathbf{D}_{p}^{H}\left(\mathbf{D}_{p} \tilde{\mathbf{R}}_{h_{p}}^{\hat{a}} \mathbf{D}_{p}^{H}+\sigma_{z}^{2} \mathbf{I}_{N_{p}}\right)^{-1} \mathbf{y}_{p} \text {. }
$$

The matrices $\tilde{\mathbf{R}}_{h ; h_{p}}^{\hat{a}}$ and $\tilde{\mathbf{R}}_{h_{p}}^{\hat{a}}$ are defined similarly as in Subsection II-A. More detailed information on the hypothesis test can be found in $[6,14,15]$. For hypothesis testing in time-variant frequency-selective channels we refer to $[6,15]$.

\section{Numerical Optimization of the Number of Hypotheses}

The optimum number of hypotheses $A_{\mathrm{opt}}$ is not known. In this section we present a framework to determine $A_{\text {opt }}$ by numerical optimization. As optimization criterion we consider the mean square channel estimation error (MSE).

For the optimization process we vary the number of hypotheses $A_{i} \in\left\{1, \ldots, A_{\max }\right\}$ and determine the number of hypotheses $A_{\text {opt }}$ that provides the minimum overall MSE. This will be explained in more detail presently. The variable $A_{\max }$ denotes the maximum number of hypotheses to be tested against and will be defined in Section III.
For each $A_{i} \in\left\{1, \ldots, A_{\max }\right\}$ we define $A_{i}$ hypotheses according to Eq. (12) and simulate $F$ frames of length $M$. For every frame $f \in\{1, \ldots, F\}$ the Doppler support of the current channel realization is drawn randomly from the uniform distribution $\mathcal{U}\left[0, \nu_{\text {Dmax }}\right]$, i.e., $\nu_{\mathrm{D}}^{f} \sim \mathcal{U}\left[0, \nu_{\text {Dmax }}\right]$. The hypothesis test, performed according to Section II-B, provides a hypothesis $\hat{a}^{f} \in\left\{1, \ldots, A_{i}\right\}$ that is used to calculate $\tilde{\mathbf{R}}_{h}^{\hat{a}^{f}}$ of the current channel realization. With (18) we obtain $\hat{\mathbf{h}}_{\text {LMMSE }}^{\hat{a}^{f}}$ and the MSE of one frame $f$ is calculated by

$$
\operatorname{MSE}^{f}=\frac{1}{M}\left\|\mathbf{h}^{f}-\hat{\mathbf{h}}_{\mathrm{LMMSE}}^{\hat{a}^{f}}\right\|^{2},
$$

where $\mathbf{h}^{f}$ denotes the current channel realization for frame $f$.

For the evaluation of the overall MSE performance we calculate the average MSE over all frames $F$. For a hypotheses size of $A_{i}$ it is calculated according to

$$
\bar{\sigma}_{A_{i}}^{\mathrm{MC}}=\frac{1}{F} \sum_{f=1}^{F} \mathrm{MSE}^{f} .
$$

The optimum number of hypotheses $A_{\mathrm{opt}}$ is the number of hypotheses that minimizes $\bar{\sigma}_{A_{i}}^{\mathrm{MC}}$, i.e.,

$$
A_{\mathrm{opt}}^{\mathrm{MC}}=\underset{A_{i} \in\left\{1, \ldots, A_{\max }\right\}}{\operatorname{argmin}} \bar{\sigma}_{A_{i}}^{\mathrm{MC}} .
$$

To assess the performance of the adaptive subspace selection algorithm for different Doppler bandwidths we introduce a second evaluation method. For this method the frames are grouped into a number of $B$ equidistant bins. Each bin $b \in\{1, \ldots, B\}$ corresponds to a Doppler support $W_{t}(b)=$ $\left(-b / B \cdot \nu_{\text {Dmax }}, b / B \cdot \nu_{\text {Dmax }}\right)$. For a channel realization $f$ to belong to the bin $b$ the Doppler support $\nu_{\mathrm{D}}^{f}$ must fulfill $(b-1) / B \cdot \nu_{\text {Dmax }}<\nu_{\mathrm{D}}^{f} \leq b / B \cdot \nu_{\text {Dmax }}$. The average MSE of a bin $b \in\{1, \ldots, B\}$ is then calculated by

$$
\overline{\mathrm{MSE}}_{b}=\frac{1}{F_{b}} \sum_{f=1}^{F_{b}} \mathrm{MSE}_{b}^{f}
$$

where $F_{b}$ is the number of frames belonging to bin $b$ and $\mathrm{MSE}_{b}^{f}$ is the MSE of the $f$-th frame that belongs to bin $b$.

\section{Simulation Results}

For the simulations we consider Monte Carlo (MC) simulations with $F=10000$ frames for each hypothesis size $A_{i}$. The time-variant flat-fading channel is assumed to have a DSD with flat support. Thus no error is made by the approximation $\mathbf{R}_{h} \approx \tilde{\mathbf{R}}_{h}$ and (8) and (11) provide the optimum dimension $D$ and $D^{(\mathcal{P})}$ for reduced-rank approximation. The maximum relative velocity between transmitter and receiver is $v_{\text {max }}=200 \mathrm{~km} / \mathrm{h}$.

For the carrier frequency and the symbol duration we assume parameters similar to the LTE downlink. We set the carrier frequency $f_{c}=2.6 \mathrm{GHz}$ and the symbol duration $T_{s}=71.43 \mu \mathrm{s}$. We simulate frames with $M=42$ symbols per frame which corresponds to three LTE subframes. This leads to $\nu_{\text {Dmax }}=0.0344$ and a time-bandwidth product (TBWP) $M \nu_{\text {Dmax }}=1.444$. The signal-to-noise ratio (SNR) was set to $10 \mathrm{~dB}$. To gain an intuitive insight on the optimal number of hypotheses the pilot distance is set to $\Delta_{t}=1$, i.e., only 
pilot symbols are transmitted. This pilot pattern is e.g. relevant for the pilot subcarriers in $802.11 \mathrm{p}$. The maximum number of hypotheses tested is $A_{\max }=100$ and the number of bins is set to $B=20$ which corresponds to a bin size of $10 \mathrm{~km} / \mathrm{h}$. In the following Fig. 1 we plot the overall average $\bar{\sigma}_{A_{i}}^{\mathrm{MC}}$ dependent on the hypothesis size $A_{i}$.

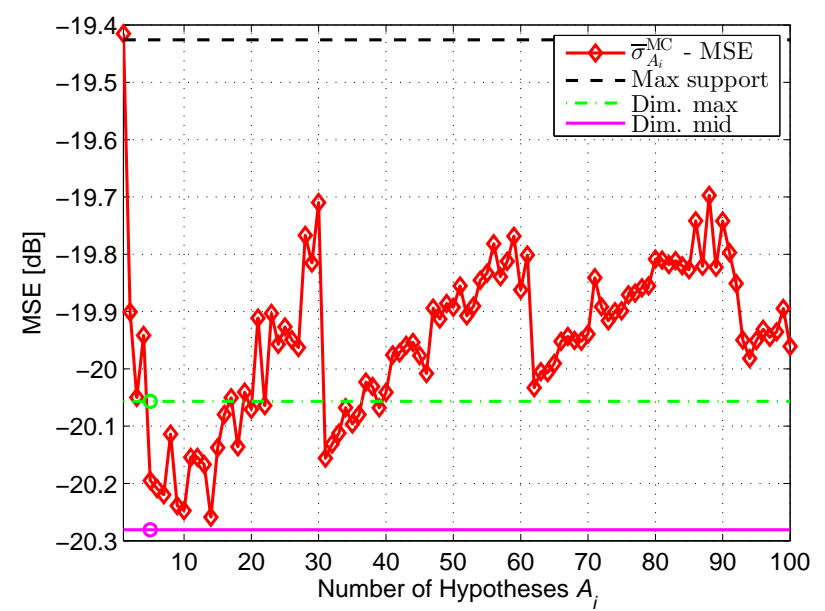

Fig. 1. $\bar{\sigma}_{A_{i}}^{\mathrm{MC}}$ vs. hypothesis size $A_{i}$ for a time-variant frequency-flat channel; $\Delta_{t}=1, \stackrel{A^{i}}{M}=42, v_{\max }=200 \mathrm{~km} / \mathrm{h}, \mathrm{SNR}=10 \mathrm{~dB}$

We observe that the overall MSE has a minimum for a hypothesis size of 14 . The minimum corresponds to the optimum number of hypotheses $A_{\text {opt }}$. A lower number $A_{i}<A_{\text {opt }}$ leads to performance degradation, whereas a higher number $A_{i}>A_{\text {opt }}$ additionally leads to an increase in receiver complexity. This is an important insight for the design of adaptive receiver structures of this kind to save complexity. The zigzag behavior of the curve is a result of the hypothesis design and of the properties of the hypothesis test.

Since the optimization process over different hypotheses sizes $A_{i}$ takes a long time we tested the performance for certain hypothesis setups which are presented schematically by horizontal lines in Fig. 1. The number of utilized hypotheses is marked by circles of the corresponding color. For the setup 'Max support' (black curve) we only consider one hypothesis with maximum Doppler support $\nu_{\text {Dmax }}$. Obviously, assuming only the maximum Doppler support for channel estimation leads to a suboptimal estimation performance. This will be discussed in more detail later on. The setup 'Dim. max' (green curve) is a specific setup that depends on the eigenvalue distribution of the autocorrelation process $\tilde{\mathbf{R}}_{h}^{(\mathcal{P})}$. We choose the hypotheses in such a way that each hypothesis has a different dimension $D^{(\mathcal{P})}$. The support of each hypothesis is the maximum support that still provides the dimension $D^{(\mathcal{P})}$. Specifically, we separate $\nu_{\text {Dmax }}$ into $A_{\text {th }}=400$ hypotheses and calculate for each hypothesis the dimension $D_{a_{\mathrm{th}}}^{(\mathcal{P})}, a_{\mathrm{th}} \in$ $\left\{1, \ldots, A_{\text {th }}\right\}$ according to (11). Depending on the eigenvalue distribution of the current hypothesis, different hypotheses may have the same dimension, i.e., $D_{i}^{(\mathcal{P})}=D_{j}^{(\mathcal{P})}, i \neq j$, $i, j \in\left\{1, \ldots, A_{\text {th }}\right\}$. The hypotheses with the same dimension are grouped. For the hypotheses set of 'Dim. max' we take the hypothesis with maximum support within a group of the same dimension. For the hypothesis setup 'Dim. mid' the Doppler support of the hypotheses is set to the middle of the support of the hypotheses of 'Dim. max'. A graphical representation of this setup is shown in Fig. 2.

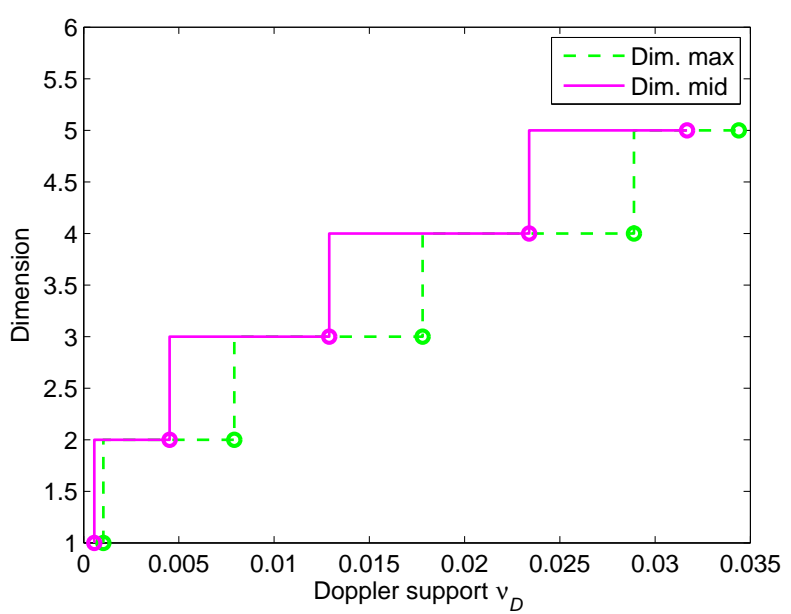

Fig. 2. Doppler support and dimension for the hypothesis setup 'Dim. max' (green) and 'Dim. mid' (magenta); $\Delta_{t}=1, M=42, v_{\max }=200 \mathrm{~km} / \mathrm{h}$, $\mathrm{SNR}=10 \mathrm{~dB}$

From Fig. 1 we see that 'Dim. mid' obtains good performance results. For a pilot spacing of $\Delta_{t}=1$ it performs similar to the optimum number of hypotheses. The performance of 'Dim. mid' depends on $\Delta_{t}$, the TBWP and SNR. It does not always perform better than the optimum number of hypotheses, however, it obtains good performance for different system parameter configurations. Considering Fig. 2 , we observe that the number of required hypotheses is only 5 which is less than $A_{\text {opt }}$. Thus 'Dim. mid' provides a good trade-off between estimation performance and complexity. For the setup only $\nu_{\text {Dmax }}, M$ and the SNR are required.

In Fig. 3 we show $\overline{\mathrm{MSE}}_{b}$ versus the Doppler bandwidth $\nu_{\mathrm{D}}$ for different hypothesis setups. We observe that the setup 'Dim. mid' performs similar to the setup with the optimum number of hypothesis. For small $\nu_{\mathrm{D}}$ adaptive subspace selection gains up to $4 \mathrm{~dB}$ compared to a hypothesis setup that only assumes $\nu_{\text {Dmax }}$. This results are similar to [6]. The gain decreases with increasing $\nu_{\mathrm{D}}$. For larger Doppler bandwidths a performance loss compared to the maximum support can be observed. This performance loss originates from the suboptimal hypothesis choice of $\hat{a}$ of the hypothesis test. For performance comparison the Wiener filter (WF) bound with exact knowledge of $\mathbf{R}_{h}$ is shown, which can be calculated according to [17, Eq. (6)-(7)].

Finally, Fig. 4 shows $\overline{\mathrm{MSE}}_{b}$ versus $A_{i}$ for different bins $b$. We observe that bins that correspond to lower speeds obtain a large performance gain when the number of hypotheses is increased. On the other hand, bins that correspond to higher speeds obtain a performance loss. Furthermore we see that depending on the bin the performance does not change significantly after passing a certain hypothesis size. This also underlines that increasing the number of hypotheses does not necessarily provide a better estimation performance.

Further simulation results, that are not provided due to space 


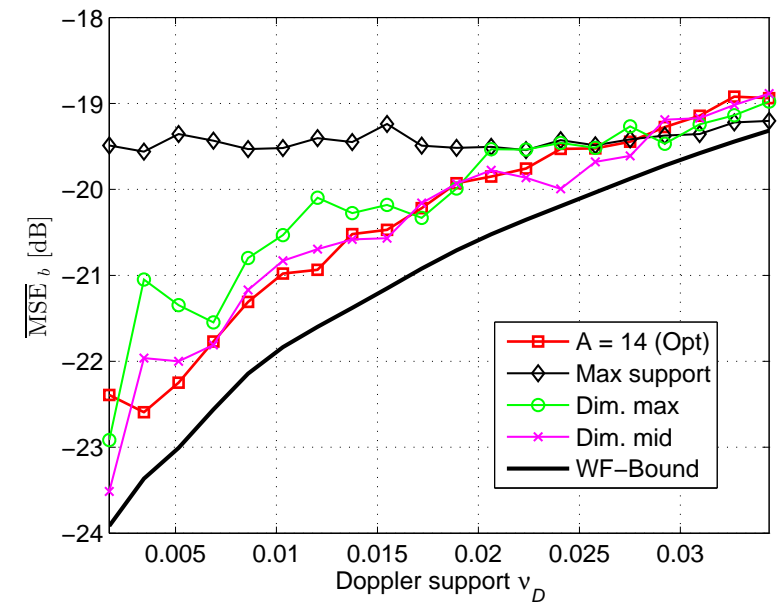

Fig. 3. $\overline{\operatorname{MSE}}_{b}$ vs. $\nu_{\mathrm{D}} ; \Delta_{t}=1, M=42, v_{\max }=200 \mathrm{~km} / \mathrm{h}, \mathrm{SNR}=10 \mathrm{~dB}$

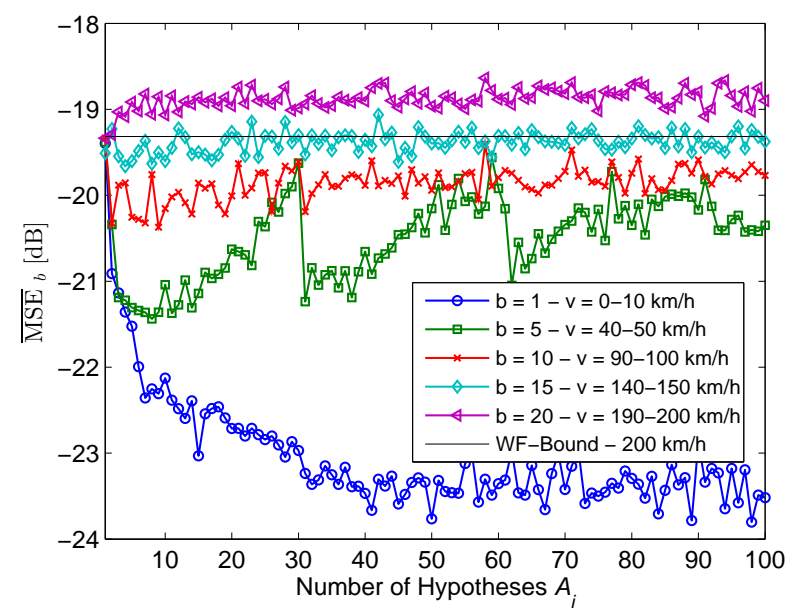

Fig. 4. $\overline{\mathrm{MSE}}_{b}$ vs. $A_{i} ; \Delta_{t}=1, M=42, v_{\max }=200 \mathrm{~km} / \mathrm{h}, \mathrm{SNR}=10 \mathrm{~dB}$

limitations suggest an increase by trend of $A_{\text {opt }}$ with increasing TBWP and SNR. For $\Delta_{t}$ no exact conclusion can yet be given.

\section{CONCLUSION}

In this paper we presented new insights on the numerical optimization of the number of hypotheses for adaptive reduced-rank channel estimation in time-variant frequencyflat fading channels. We found that the overall MSE shows a minimum for a certain hypothesis size and presented a simple hypothesis setup 'Dim. mid' that provides a good trade-off between estimation performance and complexity. For our adaptive channel estimation algorithm only the maximum Doppler support $\nu_{\text {Dmax }}$, the frame-length $M$ and the SNR are required, allowing its application for non-stationary (vehicular) channel estimation.

\section{ACKNOWLEDGMENTS}

This research was supported by the project NOWIRE funded by the Vienna Science and Technology Fund (WWTF), the project NFN SISE (S10607) funded by the Austrian Science Fund (FWF). The Austrian Competence Center FTW Forschungszentrum Telekommunikation Wien $\mathrm{GmbH}$ is funded within the program COMET - Competence Centers for
Excellent Technologies by BMVIT, BMWFJ, and the City of Vienna. The COMET program is managed by the FFG.

\section{REFERENCES}

[1] "ETSI TR 102638 - Intelligent Transport Systems (ITS); Vehicular Communications; Basic Set of Applications; Definitions."

[2] E. G. Strom, "On Medium Access and Physical Layer Standards for Cooperative Intelligent Transport Systems in Europe," Proceedings of the IEEE, vol. 99, no. 7, pp. 1183-1188, Jul. 2011.

[3] "IEEE Standard for Information Technology-Telecommunications and Information Exchange between Systems Local and Metropolitan Area Networks-Specific Requirements Part 11: Wireless LAN Medium Access Control (MAC) and Physical Layer (PHY) Specifications," IEEE Std 802.11-2012 (Revision of IEEE Std 802.11-2007), pp. 1-2793, 2012.

[4] 3GPP, "Technical Specification Group Radio Access Network; (EUTRA) and (E-UTRAN); Overall Description; stage 2," Tech. Rep., Sep. 2008. [Online]. Available: http://www.3gpp.org/ftp/Specs/ html-info/36300.htm

[5] G. Araniti, C. Campolo, M. Condoluci, A. Iera, and A. Molinaro, "LTE For Vehicular Networking: A Survey," IEEE Communications Magazine, vol. 51, no. 5, pp. 148-157, May 2013.

[6] T. Zemen and A. F. Molisch, "Adaptive Reduced-Rank Estimation of Nonstationary Time-Variant Channels Using Subspace Selection," IEEE Transactions on Vehicular Technology, vol. 61, no. 9, pp. 4042-4056, Nov. 2012.

[7] A. Molisch, F. Tufvesson, J. Karedal, and C. Mecklenbrauker, "A Survey on Vehicle-to-Vehicle Propagation Channels," IEEE Wireless Communications, vol. 16, no. 6, pp. 12-22, Dec. 2009.

[8] C. F. Mecklenbrauker, A. F. Molisch, J. Karedal, F. Tufvesson, A. Paier, L. Bernado, T. Zemen, O. Klemp, and N. Czink, "Vehicular Channel Characterization and Its Implications for Wireless System Design and Performance," Proceedings of the IEEE, vol. 99, no. 7, pp. 1189-1212, Jul. 2011.

[9] L. Bernado, T. Zemen, F. Tufvesson, A. F. Molisch, and C. F. Mecklenbrauker, "Delay and Doppler Spreads of Nonstationary Vehicular Channels for Safety-Relevant Scenarios," IEEE Transactions on Vehicular Technology, vol. 63, no. 1, pp. 82-93, Jan. 2014

[10] Z. Xu, L. Bernado, M. Gan, M. Hofer, T. Abbas, V. Shivaldova, K. Mahler, D. Smely, and T. Zemen, "Relaying for IEEE 802.11p at Road Intersection Using A Vehicular Non-Stationary Channel Model," in 2014 IEEE 6th International Symposium on Wireless Vehicular Communications (WiVeC 2014), no. 1. IEEE, Sep. 2014, pp. 1-6.

[11] T. Zemen and C. Mecklenbrauker, "Time-Variant Channel Estimation Using Discrete Prolate Spheroidal Sequences," IEEE Transactions on Signal Processing, vol. 53, no. 9, pp. 3597-3607, Sep. 2005.

[12] T. Zemen, L. Bernado, N. Czink, and A. F. Molisch, "Iterative TimeVariant Channel Estimation for 802.11p Using Generalized Discrete Prolate Spheroidal Sequences," IEEE Transactions on Vehicular Technology, vol. 61, no. 3, pp. 1222-1233, Mar. 2012.

[13] D. Slepian, "Prolate Spheroidal Wave Functions, Fourier Analysis, and Uncertainty-V: The Discrete Case," Bell System Technical Journal, vol. 57, no. 5, pp. 1371-1430, May 1978.

[14] T. Zemen, C. Mecklenbrauker, F. Kaltenberger, and B. Fleury, "Minimum-Energy Band-Limited Predictor With Dynamic Subspace Selection for Time-Variant Flat-Fading Channels," IEEE Transactions on Signal Processing, vol. 55, no. 9, pp. 4534-4548, Sep. 2007.

[15] M. Hofer and T. Zemen, "Iterative Non-Stationary Channel Estimation for LTE Downlink Communications," in 2014 IEEE International Conference on Communications Workshops (ICC). IEEE, Jun. 2014, pp. 26-31.

[16] S. Beheshti and M. Dahleh, "A New Information-Theoretic Approach to Signal Denoising and Best Basis Selection," IEEE Transactions on Signal Processing, vol. 53, no. 10, pp. 3613-3624, Oct. 2005.

[17] A. Ispas, M. Dorpinghaus, G. Ascheid, and T. Zemen, "Characterization of Non-Stationary Channels Using Mismatched Wiener Filtering," IEEE Transactions on Signal Processing, vol. 61, no. 2, pp. 274-288, Jan. 2013.

[18] X. Mestre, "Improved Estimation of Eigenvalues and Eigenvectors of Covariance Matrices Using Their Sample Estimates," IEEE Transactions on Information Theory, vol. 54, no. 11, pp. 5113-5129, 2008.

[19] L. Scharf and D. Tufts, "Rank Reduction for Modeling Stationary Signals," IEEE Transactions on Acoustics, Speech, and Signal Processing, vol. 35, no. 3, pp. 350-355, Mar. 1987. 